\title{
An update on somatostatin receptor signaling in native systems and new insights on their pathophysiology
}

\author{
Davide Cervia $^{\mathrm{a}, \mathrm{b}, *}$, Paola Bagnoli ${ }^{\mathrm{b}}$ \\ a Department of Environmental Sciences, University of Tuscia, largo dell'Università snc, blocco D, 01100 Viterbo, Italy \\ ${ }^{\mathrm{b}}$ Department of Biology, Unit of General Physiology, University of Pisa, via San Zeno 31, 56127 Pisa, Italy
}

\begin{abstract}
The peptide somatostatin (SRIF) has important physiological effects, mostly inhibitory, which have formed the basis for the clinical use of SRIF compounds. SRIF binding to its 5 guanine nucleotide-binding proteins-coupled receptors leads to the modulation of multiple transduction pathways. However, our current understanding of signaling exerted by receptors endogenously expressed in different cells/tissues reflects a rather complicated picture. On the other hand, the complexity of SRIF receptor signaling in pathologies, including pituitary and nervous system diseases, may be studied not only as alternative intervention points for the modulation of SRIF function but also to exploit new chemical space for drug-like molecules.

(C) 2007 Elsevier Inc. All rights reserved.

Keywords: Endogenous receptor; G protein; Transduction pathway; Somatotropinoma; Nervous system disease

Abbreviations: $\left[\mathrm{Ca}^{2+}\right]_{\mathrm{i}}$, intracellular $\mathrm{Ca}^{2+}$ concentration; $\mathrm{A} \beta$, amyloid- $\beta$ peptide; $\mathrm{AD}$, Alzheimer's disease; CaMKII, $\mathrm{Ca}^{2+} / \mathrm{calmodulin}^{2}$ dependent protein kinase II; cdk, cyclin-dependent protein kinase; DEP/PTP, density-enhanced protein-tyrosine phosphatase; ERK, extracellular signal-regulated protein kinases; G proteins, guanine nucleotide-binding proteins; GH, growth hormone; GIP, GPCR-interacting proteins; GPCR, G protein-coupled receptors; GRK, GPCR protein kinases; GSK, glycogen synthase protein kinase; IKK, inhibitor $\kappa B$ protein kinase; ILK, integrin-linked protein kinase; IP ${ }_{3}$, inositol trisphosphate; MAP kinases, mitogenactivated protein kinases; PDK, phosphoinositide-dependent kinase; PI3 kinase, phosphatidylinositol 3 protein kinase; PKA, cAMP-dependent protein kinase; PKC, phospholipase C-dependent protein kinase; PKG, cGMP-dependent protein kinase; PP, serine/threonine phosphatases; RGS, regulators of G-protein signaling; r-PTP, receptor-like tyrosine phosphatase; SAPK/JNK, stress-activated protein kinase/c-Jun $\mathrm{NH}_{2}$-terminal protein kinase; $\mathrm{SHP}$, $\mathrm{SH} 2$ domain-containing cytosolic tyrosine phosphatase; SRIF, somatostatin or somatotropin release inhibiting factor; sst $_{1-5}$, somatostatin receptor 1 to 5 .
\end{abstract}

\section{Contents}

1. Introduction . . . . . . . . . . . . . . . . . . . . . . 323

2. Somatostatin receptor signaling in native systems . . . . . . . . . . . . . . . . . 323

2.1. Adenylyl cyclase pathway . . . . . . . . . . . . . . . . . . . . . . 324

2.2. Guanylyl cyclase/nitric oxide pathway . . . . . . . . . . . . . . . . . . . . . . . . . . . . . . . . 324

2.3. Phospholipase C pathway . . . . . . . . . . . . . . . . . . . . . . . . . . . . . . . . 325

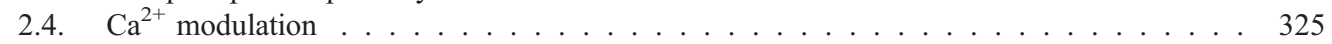

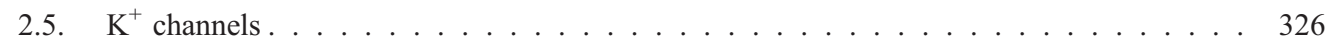

2.6. Phospholipase $\mathrm{A}_{2}$ pathway . . . . . . . . . . . . . . . . . . . 327

2.7. $\mathrm{Na}^{+} / \mathrm{H}^{+}$exchanger . . . . . . . . . . . . . . . . . . . . . . . 327

2.8. Phosphoprotein phosphatase pathway . . . . . . . . . . . . . . . . 327

2.9. Mitogen-activated protein kinase pathway . . . . . . . . . . . . . . . . 328

\footnotetext{
* Corresponding author. Department of Environmental Sciences, University of Tuscia, largo dell'Università snc, blocco D, 01100 Viterbo, Italy. Tel.: +39 0761 354070; fax: +39 0761357179 .

E-mail address: d.cervia@unitus.it (D. Cervia).
} 